\title{
Fault Identification of Rotor System Based on Classifying Time-Frequency Image Feature Tensor
}

\author{
Hui Li, ${ }^{1,2}$ Xiaofeng Liu, ${ }^{2}$ and Lin Bo ${ }^{2}$ \\ ${ }^{1}$ Chongqing College of Electronic Engineering, Chongqing 400444, China \\ ${ }^{2}$ The State Key Laboratory of Mechanical Transmission, Chongqing 400044, China \\ Correspondence should be addressed to Xiaofeng Liu; 109742210@qq.com
}

Received 6 November 2016; Revised 5 January 2017; Accepted 19 January 2017; Published 14 March 2017

Academic Editor: Zhixiong Li

Copyright (c) 2017 Hui Li et al. This is an open access article distributed under the Creative Commons Attribution License, which permits unrestricted use, distribution, and reproduction in any medium, provided the original work is properly cited.

\begin{abstract}
In the field of rotor fault pattern recognition, most of classical pattern recognition methods generally operate in feature vector spaces where different feature values are stacked into one-dimensional (1D) vector and then processed by the classifiers. In this paper, timefrequency image of rotor vibration signal is represented as a texture feature tensor for the pattern recognition of rotor fault states with the linear support higher-tensor machine (SHTM). Firstly, the adaptive optimal-kernel time-frequency spectrogram visualizes the unique characteristics of rotor fault vibration signal; thus the rotor fault identification is converted into the corresponding timefrequency image (TFI) pattern recognition. Secondly, in order to highlight and preserve the TFI local features, the TFI is divided into some TFI subzones for extracting the hierarchical texture features. Afterwards, to avoid the information loss and distortion caused by stacking multidimensional features into vector, the multidimensional features from the subzones are transformed into a feature tensor which preserves the inherent structure characteristic of TFI. Finally, the feature tensor is input into the SHTM for rotor fault pattern recognition and the corresponding recognition performance is evaluated. The experimental results showed that the method of classifying time-frequency texture feature tensor can achieve higher recognition rate and better robustness compared to the conventional vector-based classifiers, especially in the case of small sample size.
\end{abstract}

\section{Introduction}

Rotor system as an important asset exists in a wide range of industry applications and its fault identification is crucial to its design and usage. Its vibration signals are generally used to detect its healthy state to reduce the catastrophic damage and the down-time of machinery. Disk unbalance and resonance are the two main sources of rotating machinery vibration. The vibrations due to such sources will affect the system critical parts, such as bearings, gears, motor, seals, and coupling. Disk unbalance is a condition in which the center of mass of a rotating disk is not coincident with the center of rotation. Unbalance in rotor system is unavoidable and cannot be completely eliminated. Jalan and Mohanty [1] stated that, due to some reasons such as porosity in casting, nonuniform density of material, manufacturing tolerances, and gain or loss of material during operation, rotors can never be perfectly balanced in the practical applications. Oil film instability is a common nonlinear fault in a rotor-bearing system, which may bring a serious hazard to rotary machines. Liu et al. [2] also have been devoted to study the instability behaviors of rotor-bearing systems with asymmetric inertia, which are usually caused by the coupled effects of the nonlinear oil film force, unbalanced centrifugal force, journal whirl inertia force, rotor gravity, and other external load. In the compound fault of oil film instability and unbalance, the vibration signal collected from a rotor-bearing system exhibits phase/frequency coupling features which cannot be directly used to identify rotor healthy state based on the traditional data analysis methods, such as orbit portrait, FFT spectra, cepstra and time-frequency, or time-scale analysis [3].

For the improvement of reliability, safety, and efficiency of technical processes, fault pattern recognition is used as advanced supervision tools in present industries, which not only can liberate human experience evaluation but also can achieve accurate and timely fault warning. Generally speaking, there are two main steps in the fault pattern 
recognition: the first step is the feature extraction with some signal processing methods, and the second step is the fault state identification with artificial intelligence technique based on the extracted features. It is well known that time-frequency spectrogram can visualize the unique characteristics of vibration signal, based on which the fault state can be automatically identified with the time-frequency image (TFI) recognition technology. Owing to the fact that the time-frequency matrix of vibration signal has a big randomness and strong irregularity, it is not suitable to do the direct identification of fault state with the general image recognition methods such as syntactic recognition method [4], template matching method [5], and geometrical transformation method [6] in the practical fault diagnosis. Artificial intelligence technique is a good choice to realize the automatic identification of fault condition and prevents the subjective error in the operator observation. The intelligent TFI identification usually focuses on how to extract the useful features to characterize the spectrogram image. Many types of image features, such as moment invariants [7], gray statistical characteristics, textural features [8], and differential box-counting fractal dimension [9], are usually extracted for TFI autorecognition. Most of these extracted features represent the global information of TFI. But for the vibration signals of the rotor system in fault states, the characteristic signal components concentrating in some local zone of TFI would lead to nonuniform distribution of TFI characteristics. The global feature extraction of TFI would provide some unreliable identification results since the real fault characteristics are averaged out. The useful information gets lost so as to decrease the accuracy of fault identification.

In order to make up the information loss in the global feature extraction, it is necessary to segment TFI segmentation and carry out the feature extraction in different frequency bands. That is, each frequency band regions are separately processed to extract their corresponding features. In the field of fault state pattern recognition, the multidimensional features in machine learning are generally transformed to a vector representation and then processed by the classical learning algorithms operating with vectors. Although there are many ways to vectorize multidimensional data, it has been observed that such operation usually leads to significant loss of important information, since some values which were in local vicinity become differently arranged if data are arbitrarily linearized into a vector [10]. And it is necessary to reduce the dimension of time-frequency features to appropriate size with some dimensionality reduction techniques such as factor analysis, principal component analysis, independent component analysis, and singular value decomposition [1113]. But these dimensionality reduction techniques suffer the problem of overdecomposition or incomplete decomposition which might distort the feature correlations, increase architectural complexity and malfunction of classifier, and even lead to the false classification results. In a word, despite these feasible methods in vectors operating space, they do not make a full exploitation of the TFI characteristics.

In this paper, the feature tensor is introduced to characterize the TFI of rotor vibration signal, which can keep the correlation information between different frequency bands and the integrality of the TFI. However, due to the so-called curse of dimensionality and small size problems, SVM cannot handle the tensor objects effectively. For the feature tensors of TFIs, the linear support higher-tensor machine (SHTM) is used as a tensor classifier for the rotor fault identification. The SHTM is a multilinear construct learning model constructed by extending the support vector machine learning framework to tensor patterns [14]. The approach presents the supervised tensor learning framework by applying a combination of the convex optimization and multilinear operators [15]. The experimental results showed that the approach of classifying time-frequency image feature tensor can identify the rotor faults more accurately compared to the conventional feature vector classifiers. The rest of this paper is organized as follows. Section 2 provides the introduction of adaptive optimalkernel distribution. Section 3 gives texture analysis carried out on gray level cooccurrence matrix (GLCM) of TFI. Section 4 provides the theory of linear Support higher-Order tensor machine for multiclassification. Section 5 explains the flowchart of proposed method. The experimental results, discussions, and analysis are presented in Section 6. The conclusions are given in Section 7.

\section{Adaptive Optimal-Kernel Time-Frequency Distribution and TFI Segmentation}

Adaptive optimal-kernel (AOK) time-frequency transform is a suitable tool to obtain the time-frequency images of vibration signals of rotary machine. The AOK time-frequency distribution can preserve simultaneously good resolutions in time and frequency with less cross-term interferences. It is expressed as

$$
\begin{aligned}
& \operatorname{AOK}(t, f) \\
& \quad=\iint_{-\infty}^{+\infty} A(\tau, v) \phi(\tau, v) \exp [-2 \pi(v t+f \tau)] d v d \tau,
\end{aligned}
$$

where the analyzed signal is noted as $x(t), A(\tau, v)=\int_{-\infty}^{+\infty} x(t+$ $\tau / 2) x^{*}(t-\tau / 2) \exp (-j 2 \pi v t) d t$ is the ambiguity function, and $\phi(\tau, v)$ is a $2 \mathrm{D}$ radially Gaussian kernel function performed as a low-pass filter in the ambiguity domain. The optimalkernel function can be obtained by minimizing autoterm distortion by passing autoterm energy as much as possible for a kernel of fixed volume so as to suppress cross-terms. $\operatorname{AOK}(t, f)$ is used to analyze the rotor vibration signals and the analysis results are expressed in the TFI with 256 gray levels. Based on the TFIs, how to classify the vibration signals correctly corresponding to the rotor states is a typical pattern recognition problem. In the view of too many pixels in TFI, it is necessary to transform the TFI into the expected lowdimension feature space and keep the information of TFI as much as possible.

The global statistics for the whole TFI cannot describe the local details of the signal. The fault characteristic components generally concentrate in some frequency bands of TFI, not like the noises distributed in the whole plane of TFI. In order 
to get more details about fault characteristic components, the TFI is divided into some frequency band regions, as shown in

$$
\operatorname{AOK}(t, f)=\sum_{i=1}^{I} R_{i}(t, f)
$$

where $I$ is the number of TFI segmented blocks and $R_{i}(t, f)$ is the $i$ th frequency band block. The textural descriptors are extracted in each block instead of from the whole TFI.

\section{Texture Features Extraction}

For the intelligent pattern recognition of TFIs, the key point is to extract the effective features which can accurately capture the fault information of rotor system. Texture as one of the basic feature of TFI has clear meaning representing the fault information in the rotor vibration signal and can be easily calculated. Texture feature can steadily describe the spatial changes situation of the color, the gradation or the fine structure, and shape of the image with favorable rotation invariance and anti-interference capability [16, 17]. Texture is analyzed using a statistical technique based on GLCM, which use the gray level spatial correlation, firstly to construct a cooccurrence matrix according to the direction and distance between image pixels and then to extract from cooccurrence matrix meaningful statistical data as the image texture features.

For the matrix image with gray level $N_{g}$, its horizontal and vertical direction have $N_{x}$ and $N_{y}$ pixels, respectively. Given $L_{x}=\left\{1,2, \ldots, N_{x}\right\}, L_{y}=\left\{1,2, \ldots, N_{y}\right\}$, and $G=$ $\left\{0,1, \ldots, N_{g}-1\right\}$ as quantitative gray level set, the image can be expressed as $I: L_{x} \times L_{y} \rightarrow G$. Gray level cooccurrence matrix presents a pair of pixels which is on the $\left(\Delta_{x}, \Delta_{y}\right)$ direction, horizontal distance has $\Delta_{x}$ pixels, vertical distance has $\Delta_{y}$ pixels, and each has gray value probability is of $i$ and $j$, respectively. It can be expressed as $P\left(i, j, \Delta_{x}, \Delta_{y}\right)$, which is the element of gray level cooccurrence matrix. $P\left(i, j, \Delta_{x}, \Delta_{y}\right)$ is written as follows:

$$
\begin{aligned}
& P\left(i, j, \Delta_{x}, \Delta_{y}\right) \\
& \quad=\#\left\{\begin{array}{c}
((k, l),(m, n)) \in\left(L_{x} \times L_{y}\right) \times\left(L_{x} \times L_{y}\right) \\
I(k, l)=i, I\left(m+\Delta_{y}, n+\Delta_{x}\right)=j
\end{array}\right\},
\end{aligned}
$$

where $\#\{X\}$ is the elements number of set $X$. When $P\left(i, j, \Delta_{x}, \Delta_{y}\right)$ is normalized

$$
p\left(i, j, \Delta_{x}, \Delta_{y}\right)=\frac{P\left(i, j, \Delta_{x}, \Delta_{y}\right)}{G},
$$

where $G$ is normalized constant which is sum of all $P\left(i, j, \Delta_{x}, \Delta_{y}\right)$ elements. Six typical texture features can be extracted from gray level cooccurrence matrix as follows and noted as $m_{1}, m_{2}, m_{3}, m_{4}, m_{5}, m_{6}$, respectively.

(1) Angular second moment (ASM) or called energy is used to measure the uniformity of image gray level, it can be written as follows:

$$
m_{1}=\operatorname{ASM}\left(\Delta_{x}, \Delta_{y}\right)=\sum_{i, j} p\left(\left(i, j, \Delta_{x}, \Delta_{y}\right)\right)^{2} \text {. }
$$

(2) Inverse difference moment (IDM) is

$$
m_{2}=\mathrm{IDM}=\frac{\sum_{i, j} p\left(i, j, \Delta_{x}, \Delta_{y}\right)}{1+(i-j)^{2}} .
$$

(3) Contrast (CON) reflects the clear degree of image texture; rough texture has small inertial and fine texture has large inertial; it is represented as follows:

$$
m_{3}=\operatorname{CON}\left(\Delta_{x}, \Delta_{y}\right)=\sum_{i, j}(i-j)^{2} p\left(i, j, \Delta_{x}, \Delta_{y}\right) \text {. }
$$

(4) Correlation (COR) is used to describe the matrix similarity degree between the row element or the column elements. It is a measurement for gray linear.

$$
\begin{aligned}
m_{4} & =\operatorname{COR}\left(\Delta_{x}, \Delta_{y}\right) \\
& =\frac{\left[\sum_{i, j}\left(i \times j \times p\left(i, j, \Delta_{x}, \Delta_{y}\right)\right)-\mu_{x} \mu_{y}\right]}{\sigma_{x} \sigma_{y}},
\end{aligned}
$$

where, $\mu_{x}, \mu_{y}$ and $\sigma_{x}, \sigma_{y}$ are the mean value and standard deviation of $p_{x}\left(p_{x}=\sum_{i} p\left(i, j, \Delta_{x}, \Delta_{y}\right)\right)$ and $p_{y}\left(p_{y}=\sum_{i} p\left(i, j, \Delta_{x}, \Delta_{y}\right)\right)$.

(5) Variance (VAR) of sum of squares is

$$
m_{5}=\operatorname{VAR}\left(\Delta_{x}, \Delta_{y}\right)=\sum_{i, j}(i-\mu)^{2} p\left(i, j, \Delta_{x}, \Delta_{y}\right) \text {, }
$$

where $\mu$ is the mean value of $p\left(i, j, \Delta_{x}, \Delta_{y}\right)$.

(6) Entropy (ENT) shows the clutter degree of image texture. The higher the image texture consistency, the greater the entropy; otherwise, the higher the image texture disorderly degree, the smaller the entropy

$$
\begin{aligned}
& \operatorname{ENT}\left(\Delta_{x}, \Delta_{y}\right) \\
& =\sum_{i, j}-p\left(i, j, \Delta_{x}, \Delta_{y}\right) \times \log \left(p\left(i, j, \Delta_{x}, \Delta_{y}\right)\right) .
\end{aligned}
$$

\section{Linear Support Higher-Order Tensor Machine for Multiclassification}

Tensor presentation has the advantage of preserving intrinsic structure of dataset, such as the relationships between dimensions within the same order and the relationships of dimensions in the different orders. Due to the so-called curse of dimensionality and the small sample size problem, the SVM model based on vector space cannot directly deal with tensor patterns. So the SHTM based on the SVM and tensor rank-order decomposition TVM is applied to classify the multidimensional features in rotor fault states. The SHTM overcomes the disadvantages of local minimal problem and nonconvex optimization problem in conventional supervised tensor learning framework.

Given a training set of $M$ pairs of samples $\left\{X_{m}, y_{m}\right\}_{m=1}^{M}$, where $X_{m}=\mathfrak{R}^{I_{1} \times I_{2} \times \cdots \times I_{N}}$ is the input data and $y_{m} \in\{-1,1\}$ 
is the corresponding class labels of $X_{m}$, the SHTM model for binary classification is in the following:

$$
\begin{array}{ll}
\min _{\mathbf{W}, b, \xi} & J(\mathbf{W}, b, \xi)=\frac{1}{2}\|\mathbf{W}\|_{F}^{2}+C \sum_{m=1}^{M} \xi_{m} \\
\text { s.t. } & y_{m}\left(\left\langle\mathbf{W}, X_{m}\right\rangle+b\right) \geq 1-\xi_{m}, \quad m=1, \ldots, M \\
& \xi_{m} \geq 0 \\
& m=1, \ldots, M
\end{array}
$$

where $\mathbf{W}$ is the weight tensor of the hyperplane, $b$ is the bias, $\xi_{m}$ is the error of the $m$ th training sample, and $C$ is the tradeoff between the classification margin and misclassification error. For the Lagrangian function of the optimization problem (11), noted as $L(W, b, a, \beta, \xi)$, let the partial derivative operations on $L(W, b, a, \beta, \xi)$ with respect to $W, b$, and $\xi_{m}$ be zeroes, respectively; we have the optimization problem of (11) as follows:

$$
\begin{aligned}
\min _{\alpha} & \frac{1}{2} \sum_{i, j=1}^{M} \alpha_{i} \alpha_{j} y_{i} y_{j}\left\langle X_{i}, X_{j}\right\rangle-\sum_{m=1}^{M} \alpha_{m} \\
\text { Subject to } & \sum_{m=1}^{M} \alpha_{m} y_{m}=0 \\
& 0 \leq \alpha_{m} \leq C, m=1, \ldots, M,
\end{aligned}
$$

where $\alpha_{m}$ are the Lagrange multipliers and $\left\langle X_{i}, X_{j}\right\rangle$ are the inner products of $X_{i}$ and $X_{j}$.

Let the rank-one decomposition of $X_{i}$ and $X_{j}$ be $X_{i} \approx$ $\sum_{r=1}^{R} \mathbf{x}_{i r}^{(1)} \circ \mathbf{x}_{i r}^{(2)} \circ \cdots \circ \mathbf{x}_{i r}^{(N)}$ and $X_{j} \approx \sum_{r=1}^{R} \mathbf{x}_{j r}^{(1)} \circ \mathbf{x}_{j r}^{(2)} \circ \cdots \circ \mathbf{x}_{j r}^{(N)}$, respectively; then the inner product of $X_{i}$ and $X_{j}$ is calculated as follows:

$$
\begin{aligned}
& \left\langle X_{i}, X_{j}\right\rangle \\
& \quad \approx \sum_{p=1}^{R} \sum_{q=1}^{R}\left\langle\mathbf{x}_{i p}^{(1)}, \mathbf{x}_{j q}^{(1)}\right\rangle\left\langle\mathbf{x}_{i p}^{(2)}, \mathbf{x}_{j q}^{(2)}\right\rangle \cdots\left\langle\mathbf{x}_{i p}^{(N)}, \mathbf{x}_{j q}^{(N)}\right\rangle .
\end{aligned}
$$

Substituting (14) into (12), then the class label of a testing example $X$ is predicted as follows:

$$
y(X)=\operatorname{sign}\left(\sum_{m=1}^{M} \sum_{p=1}^{R} \sum_{q=1}^{R} \alpha_{m} y_{m} \prod_{n=1}^{N}\left\langle\mathbf{x}_{m p}^{(n)}, \mathbf{x}_{q}^{(n)}\right\rangle+b\right),
$$

where $\mathbf{x}_{m p}^{(n)}$ and $\mathbf{x}_{q}^{(n)}$ are the elements of the rand-one decomposition of $X_{m}$ and $X$, respectively. So the normal hyperplane can be expressed as a linear combination of training samples in tensor space.

Like the $L$-class classification problem of SVM, the oneagainst-one support tensor machine needs to construct $L(L-$ 1)/2 binary classification SHTM models where each one is trained on data points from two classes. The class label of a testing example $X$ can be predicted by applying majority voting strategy; that is, the vote counting takes into account the outputs of all binary classifiers. If $X$ belongs to the $i$ th class, then the $i$ th class gets one vote; otherwise the $j$ th class gets one vote; $X$ is labeled by the class with the most votes. The differences of SHTM versus SVM are as follows:

(1) SVM forces a tensor into vectors as input, while SHTM adopts a more compact tensor representations, that is, $R$ rank-one tensor as input, which not only has the capability for capturing structural information of features, but also avoids the so-called curse of dimensionality and small size sample problems in vector classification of SVM.

(2) The decision function of vector-based SVM algorithm is in the linear form and determined by weight vector and the bias in hyperplane of vector space, while the decision function of SHTM algorithm is in the multilinear form and determined by weight tensor $\mathbf{W}$ and the bias in the hyperplane of tensor space. It is more convenient to construct a maximal separable classifier between two classes or transform tensor data into the separable feature space with the tensor decomposition and transform.

(3) When the number of training points is relatively small, it is easy to show the overfitting phenomenon in the processing of SVM learning, because it is difficult to reduce the parameter vectors in vectorbased machine learning methods. But, for the SHTM, the number of parameters can be reduced by the decomposition of the parameter tensor and the low rank replacement techniques, so as to avoid the overfitting phenomenon in high dimension and small size sample.

(4) For a set of tensor samples $\left\{X_{m} \in R^{I_{1} \times I_{2} \times \cdots \times I_{N}}, y_{m}\right\}_{m=1}^{M}$, SVM requires $O\left((M+1) \prod_{n=1}^{N} I_{n}+1\right)$ memory space, while SHTM only requires $O\left((M+1) R \sum_{n=1}^{N} I_{n}+\right.$ 1) memory space, where $R$ is the rank of tensor input. The computational complexity of SVM is $O\left(M^{2} \prod_{n=1}^{N} I_{n}\right)$; the computational complexity of SHTM is $O\left(M^{2} R^{2} \sum_{n=1}^{N} I_{n}\right)$. It is clear that that SHTM is more efficient than SVM.

\section{Proposed Method}

As mentioned previously, some useful characteristic information about rotor fault might be lost in the uniform calculation of global TFT texture features. The texture features are extracted in each subregion of TFI by segmenting frequency band. These features in the different frequency band region form a multidimensional array. The multidimensional features in machine learning are generally are firstly transformed to a vector representation and then processed by the classical learning algorithms operating with vectors. However, such multi-to-one-dimensional transformations usually lead to loss of important information. So the features are formed to a tensor preserving data dimensionality complex structure. The feature tensor can capture structural information relations among the texture feature values in different frequency bands. It has been clarified in Section 3 that the SHTM is the 
generalization of the standard linear SVM to tensor patterns into tensor space and efficient for the classification of feature tensors. Therefore, the tensor rank-one decomposition is performed on the feature tensors which are processed by the SHTM for the classification of rotor fault states. The proposed method is explained with the following steps.

Step 1. Collect the vibration signal of the rotor-bearing system in normal and fault conditions.

Step 2. Perform the time-frequency transform for each vibration signal sample, and their time-frequency distributions can be obtained and expressed as the corresponding TFIs.

Step 3. Optimally segment each TFI is into $I$ frequency band zones which are taken as $I$ subimages $R_{i}(t, f), i=1,2, \ldots, I$.

Step 4. Process TFI zone $R_{i}(t, f)$ with gray level, and its GLCM is calculated as $\operatorname{GLCM}_{R_{i}}, i=1,2, \ldots, I$. The texture features are calculated in each GLCM, which forms the feature vector $\mathbf{x}_{R_{i}}=\left[x_{1}^{R_{i}}, x_{2}^{R_{i}}, \ldots, x_{J}^{R_{i}}\right], j=1,2, \ldots, J . x_{j}^{R_{i}}$ represents the $j$ th texture feature of $\mathrm{GLCM}_{R_{i}}$.

Step 5. In order to preserve data dimensionality and allow for processing of complex structures, the texture features are represented as a synthetic feature tensor shown in (16) according to the sequence of frequency band blocks.

$$
X^{I \times J}=\left[\begin{array}{c}
\mathbf{x}_{R_{1}} \\
\vdots \\
\mathbf{x}_{R_{i}} \\
\vdots \\
\mathbf{x}_{R_{I}}
\end{array}\right]=\left[\begin{array}{ccccc}
x_{1}^{R_{1}} & \ldots & x_{j}^{R_{1}} & \ldots & x_{J}^{R_{1}} \\
\vdots & \ddots & & & \vdots \\
x_{1}^{R_{i}} & & x_{j}^{R_{i}} & & x_{J}^{R_{i}} \\
\vdots & & & \ddots & \vdots \\
x_{1}^{R_{I}} & & x_{j}^{R_{I}} & & x_{J}^{R_{I}}
\end{array}\right],
$$

Here $X^{I \times J}$ is the second-order tensor for the SHTM input.

Step 6. In view of the spatial distribution characteristics of frequency components, the absolute values of texture feature cannot reflect effectively the differences between the TFI blocks. To facilitate a fair comparison, each column value of $X^{I \times J}$ is normalized; that is, $x_{j}^{R_{i}^{\prime}}=x_{j}^{R_{i}} / \sum_{i=1}^{I} x_{j}^{R_{i}}$; then the normalized feature tensor $\widehat{X}^{I \times J}$ is gotten.

Step 7. The normalized feature tensor $\widehat{X}^{I \times J}$ (noted as is $\left.X_{p}\right)$ is decomposed into $R$ rank-1 tensor. The $R$ rank-1 decomposition for $X_{p}$ is to find the output vector $\mathbf{u}_{r}$ and $\mathbf{v}_{r}$ $(1 \leq r \leq R)$ make $X_{p} \approx \sum_{r=1}^{R} \mathbf{u}_{r} \circ \mathbf{v}_{r}$. $\mathbf{u}_{r}$ and $\mathbf{v}_{r}(1 \leq r \leq R)$ make $X_{p} \approx \sum_{r=1}^{R} \mathbf{u}_{r} \circ \mathbf{v}_{r}$. With the initial values of $r, \mathbf{u}_{r}^{0}$, and $\mathbf{v}_{r}^{0}$, set $\mathbf{u}_{r}^{t+1}=X_{p} \times_{2} \mathbf{v}_{r}^{t}, \mathbf{v}_{r}^{t+1}=X_{p} \times_{2} \mathbf{u}_{r}^{t}$ and normalize $\mathbf{u}_{r}^{t+1}, \mathbf{v}_{r}^{t+1}$. Supposing

$$
\left\|\left(\mathbf{u}_{r}^{t+1}, \mathbf{v}_{r}^{t+1}\right)-\left(\mathbf{u}_{r}^{t}, \mathbf{v}_{r}^{t}\right)\right\|_{F}^{2} \leq \varepsilon
$$

where $\varepsilon$ is the decomposing control threshold, $\mathbf{u}_{r}$ and $\mathbf{v}_{r}$ would be the outputs of the $R$ rank-1 decomposition. Otherwise, $X_{p}=X_{p}-\mathbf{u}_{r}^{t+1} \circ \mathbf{v}_{r}^{t+1}, r=r+1$, and repeat (17).

Step 8 . In the same way, the $R$ rank-1 tensor of training sample $X_{m}$ is obtained $X_{m} \approx \sum_{r=1}^{R} \mathbf{s}_{r} \circ \mathbf{t}_{r}$. The inner product of $X_{p}$ and $X_{m}$ is calculated as

$$
\begin{aligned}
\left\langle X_{p}, X_{m}\right\rangle & =\left\langle\sum_{r=1}^{R} \mathbf{u}_{r} \circ \mathbf{v}_{r}, \sum_{r=1}^{R} \mathbf{s}_{r} \circ \mathbf{t}_{r}\right\rangle \\
& =\sum_{l=1}^{R} \sum_{n=1}^{R}\left\langle\mathbf{u}_{l}, \mathbf{s}_{n}\right\rangle\left\langle\mathbf{v}_{l}, \mathbf{t}_{n}\right\rangle .
\end{aligned}
$$

Substituting (18) into (12), the sequential minimal optimization algorithm is used to find the optimal tradeoff parameter in the SHTM.

Step 9. Based on the inner product in (18), the rotor states classification result is obtained according to (15) and its classification accuracy is evaluated and compared.

\section{Applications in the Rotor System Fault Identification}

Figure 1 shows the experiment set-up used in this work. The test rig consists of motor, shown on the right of the photograph, driving a rigid cylindrical shaft supported by two cylindrical journal bearings, with a supporting journal bearing on the right end near the driving motor and an oil film journal bearing at the left end for simulating oil film instability faults. Two discs are mounted on the shaft, with one at the mid-plane between the two bearings and the other near the left oil film bearing. The shaft is not of uniform diameter throughout but has two portions with different diameters. One short portion of diameter $24 \mathrm{~mm}$ rotates in the left oil film journal bearing and has a length of $40 \mathrm{~mm}$, just slightly longer than the length of the bearing. The remainder of the shaft has a diameter of $12 \mathrm{~mm}$ and a length of $480 \mathrm{~mm}$ and has its other end rotating in the right supporting bearing. Two proximity eddy current sensors are mounted, as shown in the figure, just to the right of the center disc, to measure the horizontal and vertical vibrations of the rotor at that position. In the experiment, a small mass is attached in the rotating disc to simulate an unbalance condition. Using the test bench described, the experiments under different rotational speeds with various artificially simulated faults are conducted to verify the practical validity of the proposed method. The data sampling frequency is $2048 \mathrm{~Hz}$. The experiment details are listed in Table 1.

Considering the different rotor-bearing states, there are totally 8 running states. Moreover, the data corresponding to each rotor state is equally divided into two parts: one for reference data and the other for test data. Furthermore, for each rotor state, the reference and test data are again partitioned into some equal time-interval subsignals with the time duration $0.8 \mathrm{~s}$. There are totally 400 vibration data for 8 bearing train states, about 50 vibration signals for each state. 


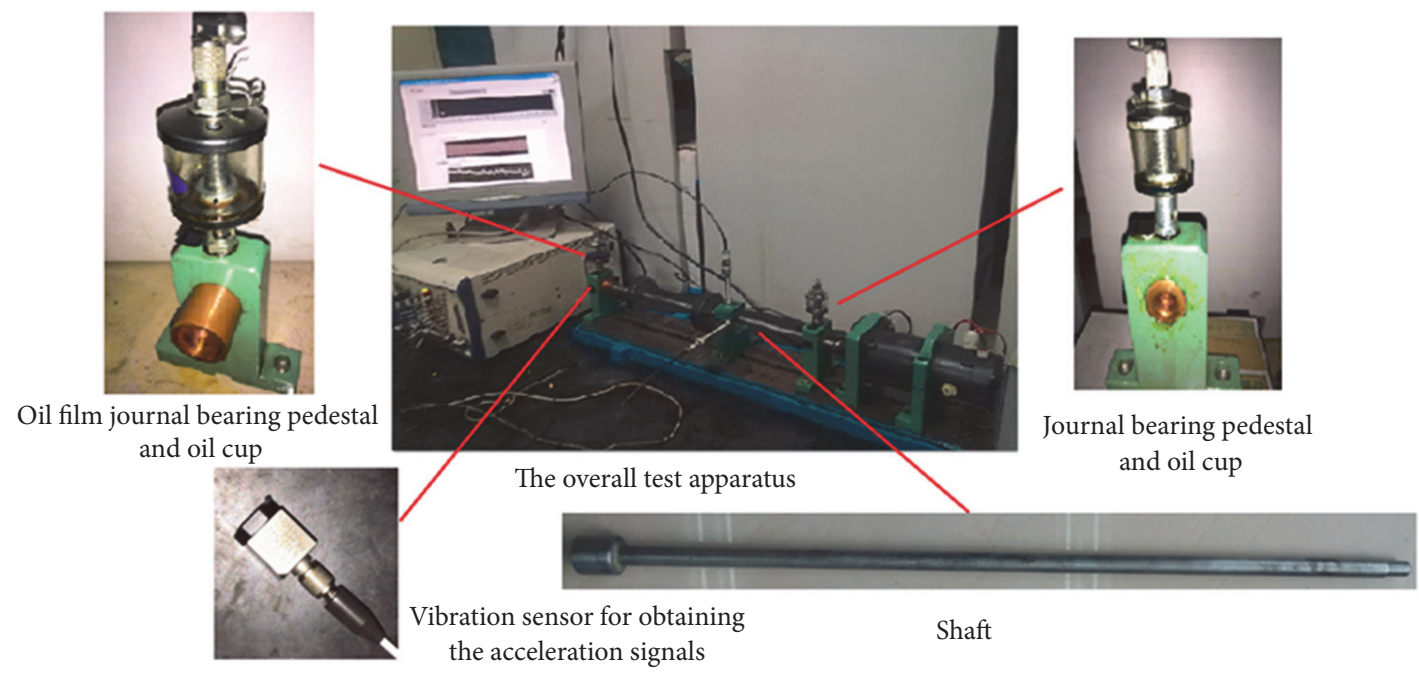

FIgURE 1: Test rig and its main components.

TABLE 1: System operation states at different operation speeds.

\begin{tabular}{|c|c|c|c|c|c|c|c|c|}
\hline Experiment & 1 & 2 & 3 & 4 & 5 & 6 & 7 & 8 \\
\hline Class code & (a) & (b) & (c) & (d) & (e) & (f) & (g) & (h) \\
\hline $\begin{array}{l}\text { Operating } \\
\text { speed/rpm }\end{array}$ & 2000 & 3000 & 5500 & 6200 & 2000 & 3000 & 5500 & 6200 \\
\hline $\begin{array}{l}\text { Observed state } \\
\text { of operation }\end{array}$ & Normal & $\begin{array}{l}\text { Resonance at first } \\
\text { critical frequency }\end{array}$ & Oil whirl & Oil whip & Unbalance & $\begin{array}{l}\text { Resonance with } \\
\text { unbalance mass }\end{array}$ & $\begin{array}{c}\text { Oil whirl with } \\
\text { unbalance mass }\end{array}$ & $\begin{array}{c}\text { Oil whip with } \\
\text { unbalance }\end{array}$ \\
\hline
\end{tabular}

After time-frequency analysis, about 50 TFIs for each state are obtained.

Firstly, AOK time-frequency transform is performed on every sample. Figure 2 depicts the AOK distributions of the rotor vibration signals in different states. The TFIs at 3000 rotating speed with and without unbalance mass are very similar, as shown in Figures 2(b) and 2(f) where the main frequency components are almost the same. The same case occurs in Figures 2(a), 2(e), 2(c), and 2(g). Therefore, it is not easy to identify the rotor states directly according to the TFIs. It is very important to perform a quantitative analysis for accurate TFI pattern classification.

Each TFI is partitioned into 5 regions $\left(R_{i}(t, f), i=\right.$ $1,2, \ldots, 5)$ with equal frequency band $200 \mathrm{~Hz}$. The GLCM is calculated for each region $R_{i}$. Based on $R_{i}$ the corresponding texture feature vectors are obtained and noted as $M_{i}=$ $\left[m_{1}^{R_{i}}, m_{2}^{R_{i}}, \ldots, m_{6}^{R_{i}}\right],(i=1,2, \ldots, 5)$, where $m_{1}^{R_{i}}, m_{2}^{R_{i}}, \ldots, m_{6}^{R_{i}}$, respectively, presents ASM, IDM, CON, COR, VAR, and ENT of TFI region $R_{i}$ in the $i$ th frequency band. To present the differences between the frequency components under each conditions, $M_{i}$ is arranged in the sequence of frequency bands to construct the tensor $M=\left[M_{1}, M_{2}, M_{3}, M_{4}, M_{5}\right]^{T}$. To a fair comparison, the column vectors for each texture feature are normalized, respectively. In order to clearly display the difference of features in eight rotor states, the texture feature tensors are mapped into the color lattices. Figure 3 provides the feature tensors of four individual signals selected from each state sample set texture tensors. It is clear that the feature tensors for the same type of rotor state are very similar, but for different fault types the obvious differences exist in the color lattices. Therefore, the feature tensors have good clustering and classifying, which can effectively represent rotor working conditions and be used to do fault states recognition.

The normalized feature tensors are put into the SHTM for classification. The classification results of the 8 rotor states are shown in Table 2. Table 2 illustrates that, when the feature tensors are used as input for the SHTM, 8 types of rotor state samples can be effectively distinguished. With the tensor features in the states "(b)"; “(c)"; "(g)"; and "(h)" (noted as in Table 1) the SHTM exhibits the best results and the recognition rate reach $100 \%$. The recognition rates of "(a)," “(b)," “(e)," and “(f)” state are 99.48\%, 97.81, 99.73\%, and $98.95 \%$, respectively. The total recognition rate is $99.5 \%$. The classification results indicate that the rotor condition can be effectively described using the texture tensors and accurately identified with the SHTM.

In order to investigate the robustness of the proposed method to the selection of TF transform, the classification accuracies based on short-time Fourier transform (STFT), Stransform (S-T), continuous wavelet transform (CWT), and AOK in the case of different segment number are compared in Figure 4. It is clear in Figure 4, whatever the segment number is, the recognition rates with different time-frequency distributions are very close with enough training samples. Comparatively speaking, AOK time-frequency transform has 


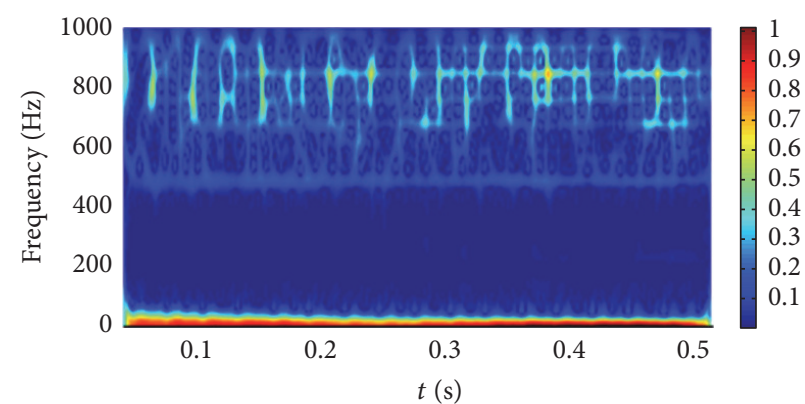

(a) Normal state

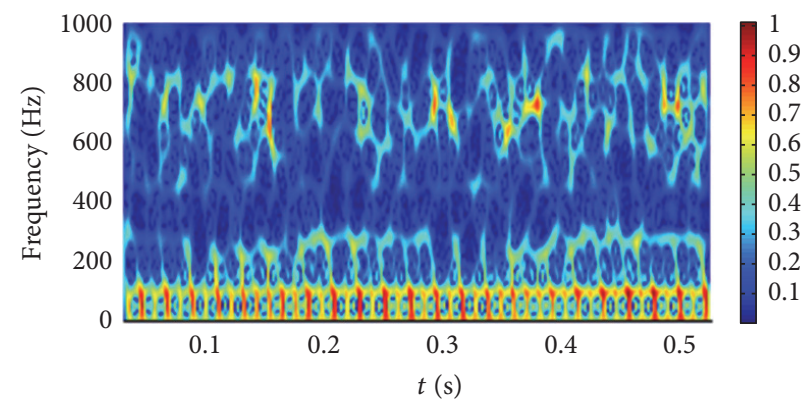

(c) Oil whirl

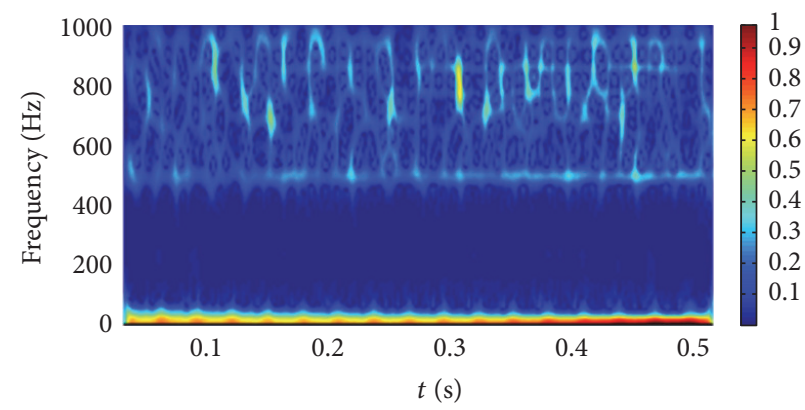

(e) Unbalance

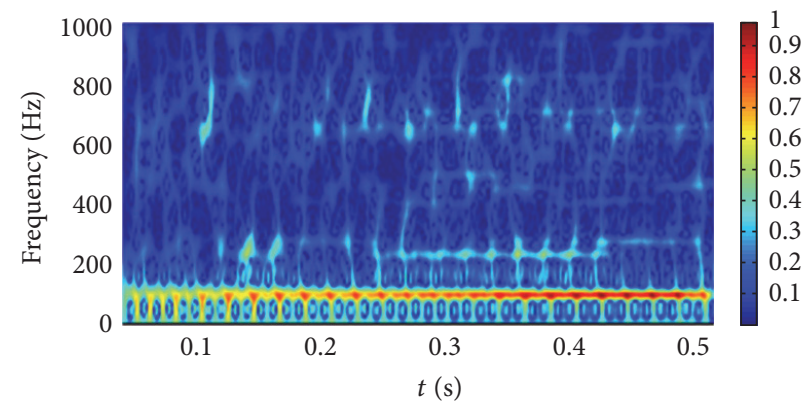

(g) Oil whirl with unbalance

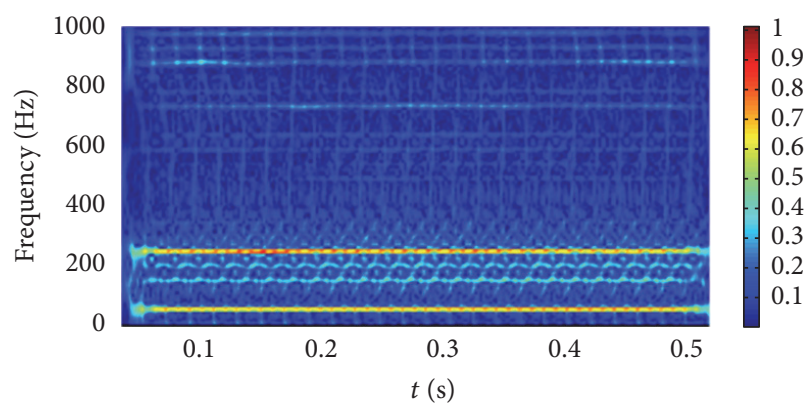

(b) Resonance at first critical frequency

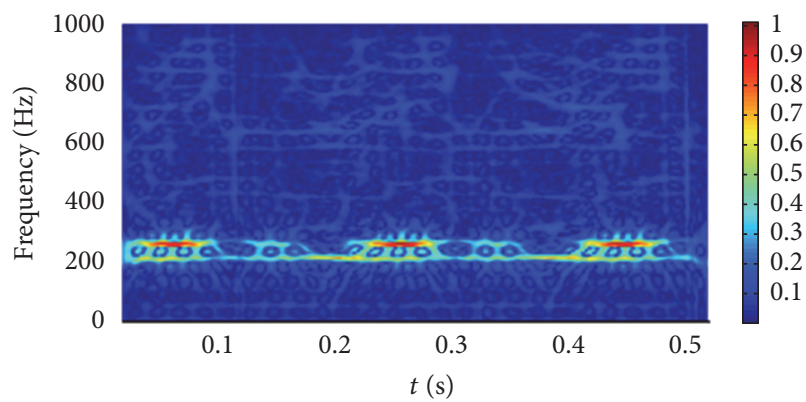

(d) Oil whip

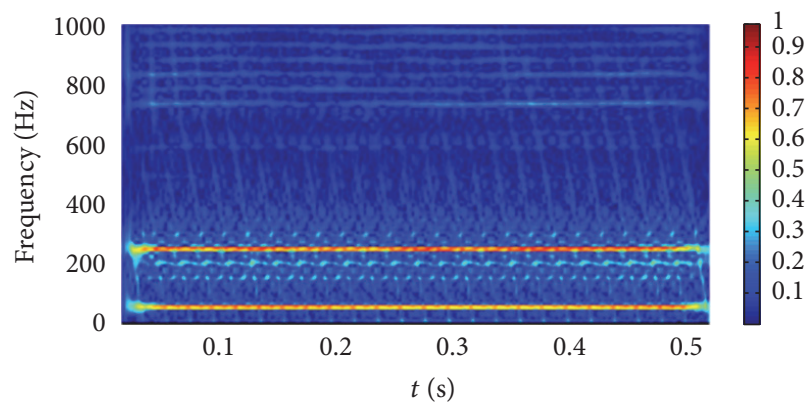

(f) Resonance with unbalance

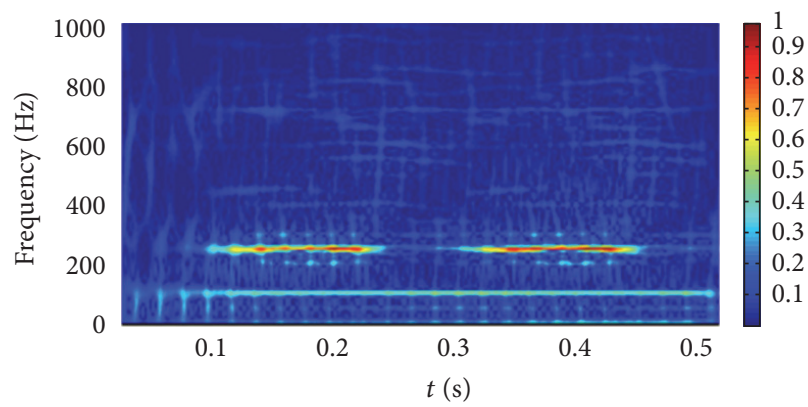

(h) Oil whip with unbalance

FIGURE 2: AOK time-frequency spectrogram for each sample vibration.

a good stability in the classification even with small sample data. That is, the proposed method has a good robustness to the selection of time-frequency distribution.

At the same time, in order to investigate the effect of segment number on the classification preciseness, the state recognition rates in the cases of different segmentations are compared with each other, as shown in Figure 5. According to Figure 5, with enough training samples, the recognition rates are nearly approaches to the same value $100 \%$ in all the cases of segmentations. In the case of the big size sample, the proposed method is robust to the segment number of TFI. In the case of the small size sample below 60, the optimal segment number is 5 , and the corresponding recognition rate reaches $99.96 \%$ with 30 training samples. In general, the segment number of TFI has a good robustness to the fault pattern recognition of rotor system. 

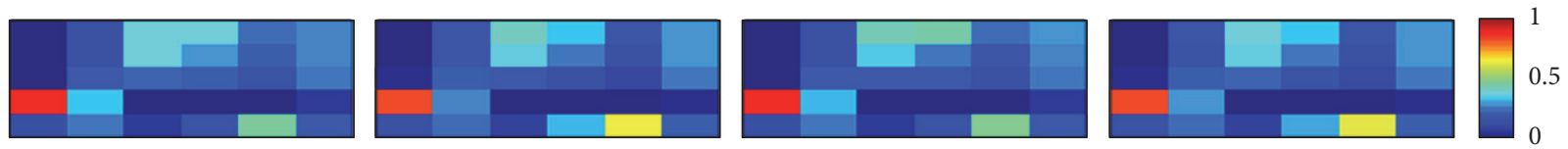

(a) Normal state
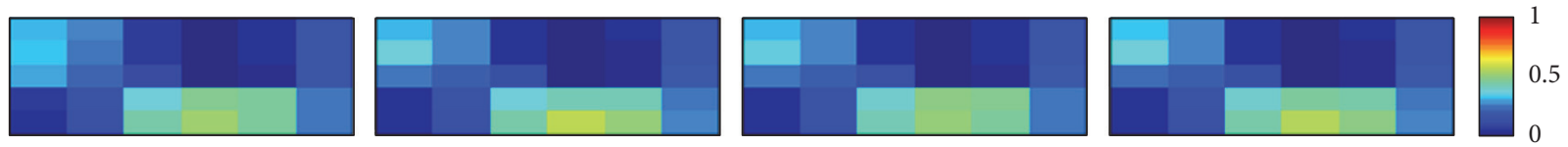

(b) Resonance at first critical frequency
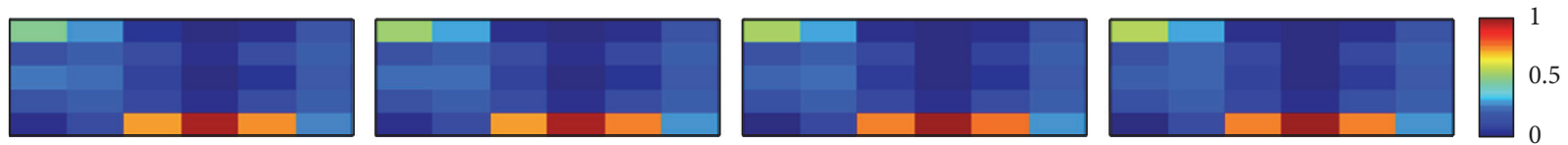

(c) Oil whirl
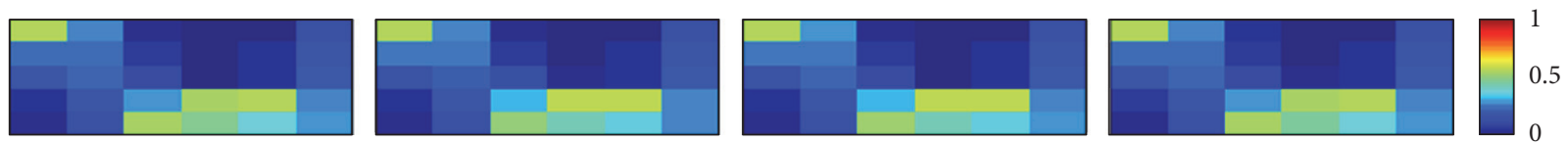

(d) Oil whip
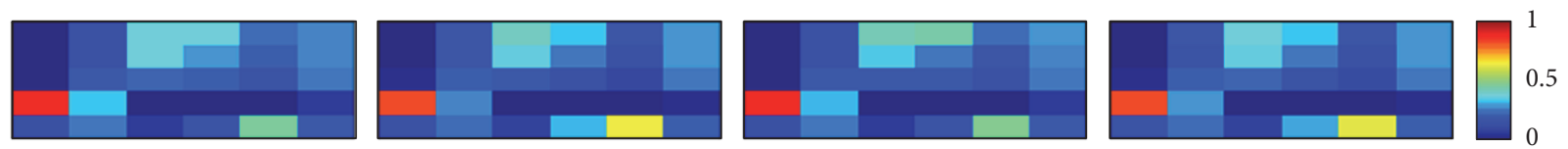

(e) Unbalance
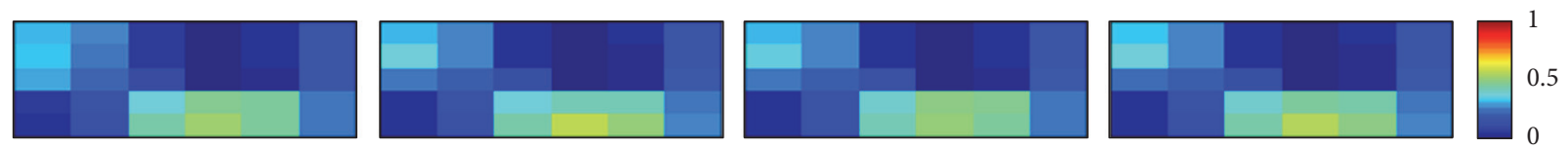

(f) Resonance with unbalance
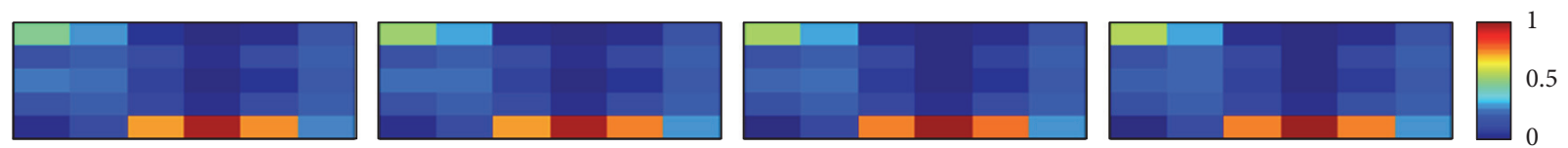

(g) Oil whirl with unbalance
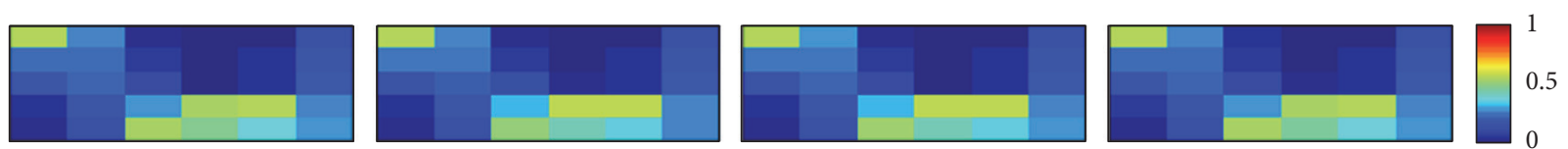

(h) Oil whip with unbalance

FIgURE 3: Texture feature tensors of TFI under 8 rotor states.

TABLE 2: Recognition results of rotor states.

\begin{tabular}{lcccc}
\hline State & Test sample number & Right recognized results & Wrong recognized results & Recognition rate \\
\hline (a) & 387 & 385 & 2 & $99.48 \%$ \\
(b) & 382 & 374 & 8 & $97.91 \%$ \\
(c) & 362 & 362 & 0 & $100 \%$ \\
(d) & 375 & 375 & 0 & $100 \%$ \\
(e) & 377 & 376 & 1 & $99.73 \%$ \\
(f) & 381 & 377 & 4 & $98.95 \%$ \\
(g) & 369 & 369 & 0 & $100 \%$ \\
(h) & 367 & 367 & 0 & $100 \%$ \\
\hline Total number & 3000 & 2985 & 15 & $\mathbf{9 9 . 5 0 \%}$ \\
\hline
\end{tabular}




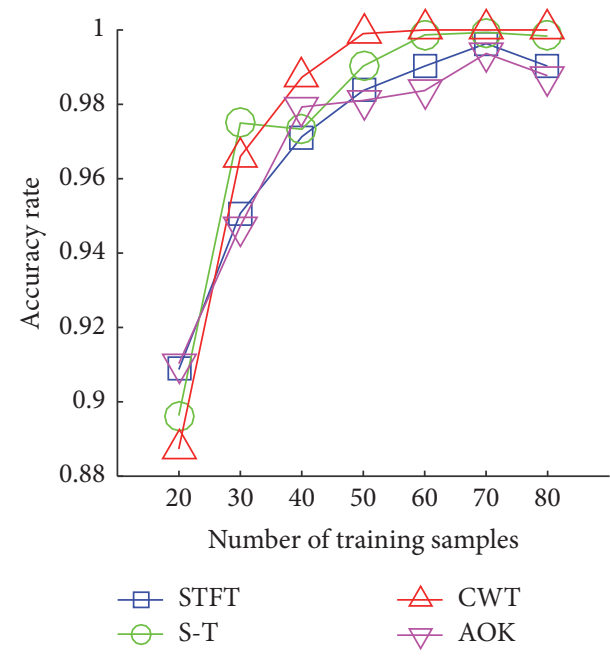

(a) $N=2$

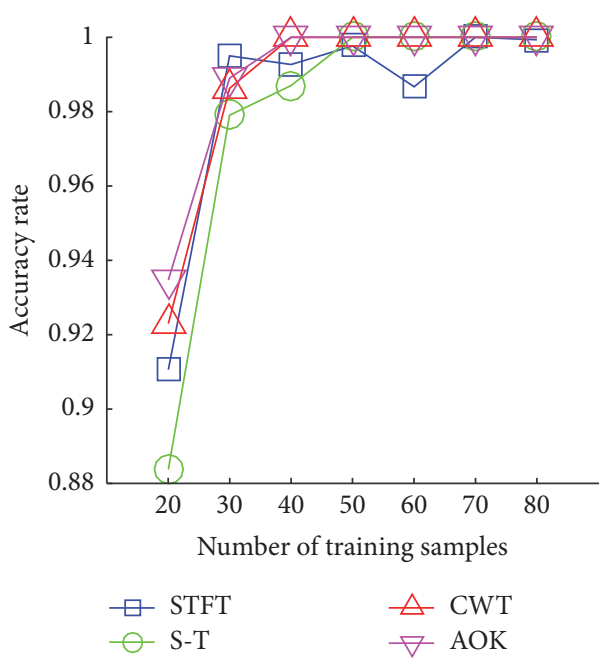

(c) $N=4$

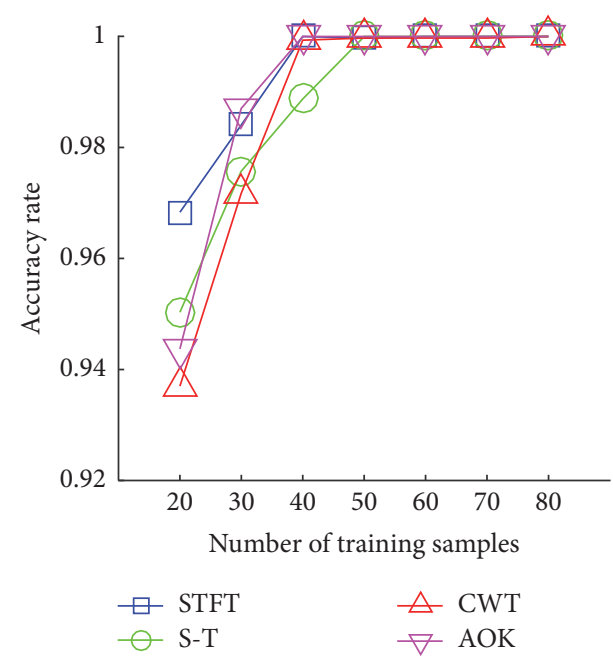

(e) $N=6$

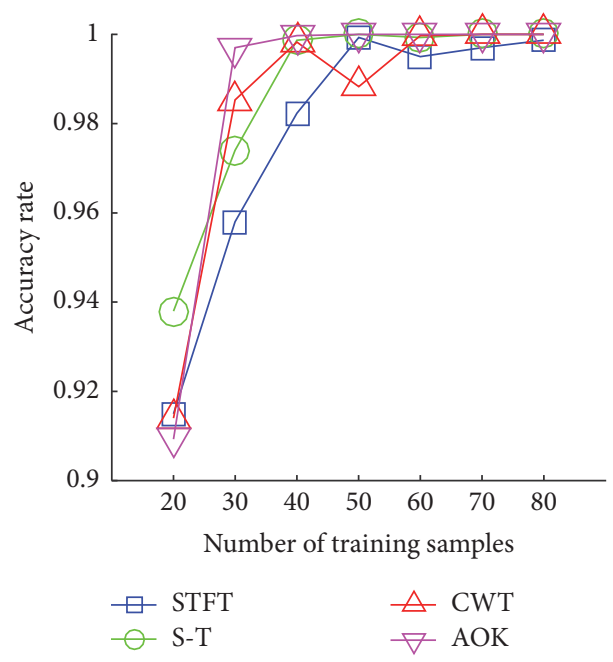

(b) $N=3$

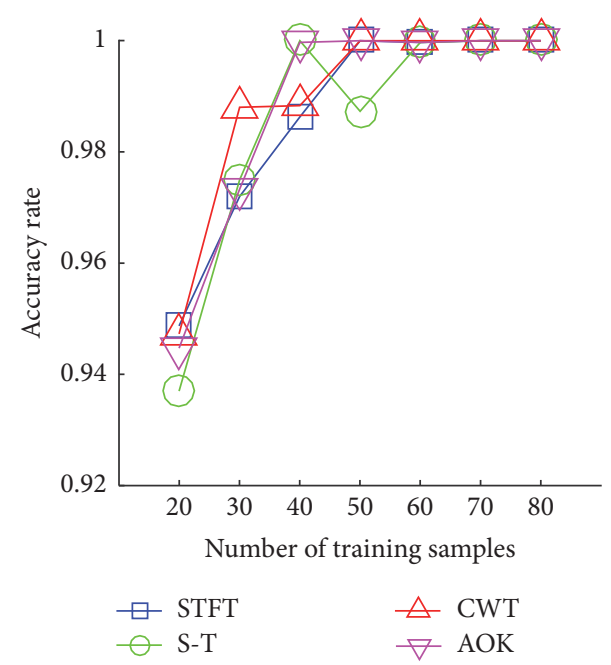

(d) $N=5$

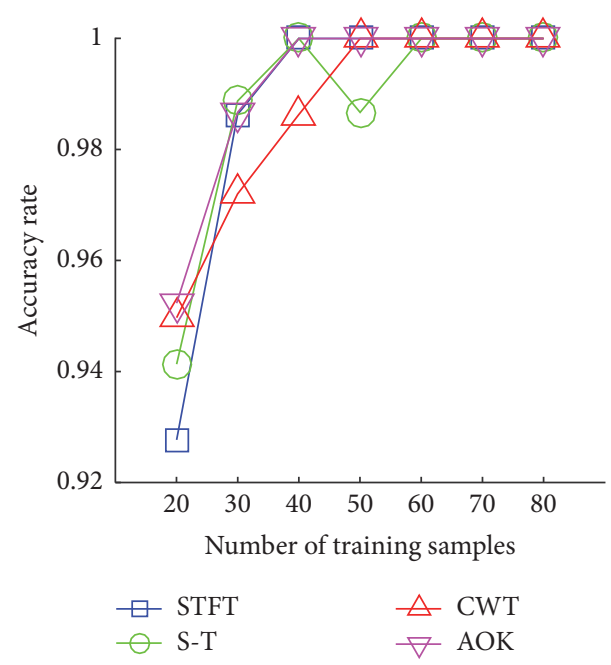

(f) $N=7$

FIGURE 4: Recognition rate comparison between different time-frequency methods. 


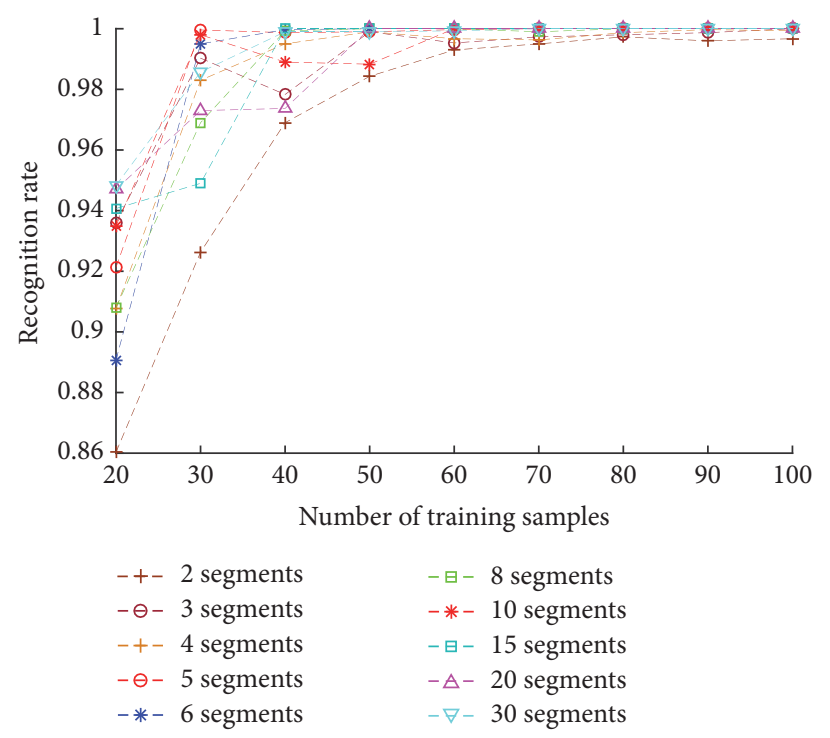

FIGURE 5: Comparison of recognition rates with different segment number.

To further evaluate the performance of proposed method, we also used the same training and test samples to carry out the SVM and BP neural network identification test in the case of different TF segmentation. For the classifiers SVM and BP under the multiple segments, the input feature vectors are constructed by stacking the texture features of each TFI subregion. The performances of each classifier are compared in Figure 6, where the number $(1,4,5)$ denotes the number of segment and "Number \& classifier" denotes the classifier in the case of the corresponding segment number. When the segment number is set as 1, the global texture features of TFI are extracted. From the Figure 6, the accuracy curve of " $N=5$ and STM" is completely above the other curves and very close to the curve of " $N=4$ and STM." It is clear that the feature tensor-based SHTM performs better than the vectors-based BP or SVM. The curves of " $N=1$ and BP" and "1 and SVM" are totally below the other curves. It is verified that using the hierarchical texture tensor greatly enhances the classification accuracy compared to using the global features.

\section{Conclusions}

In this paper, a novel approach for rotor system fault pattern recognition is proposed based on the SHTM and the timefrequency hierarchical texture feature tensor. The contributions of this paper include the following:

(1) In order to keep the correlation and interdependence between the TF characteristics in different frequency bands, hierarchical feature extraction is performed on the TFI fragment in different frequency bands. The features in each frequency band are extracted separately and treated differently. The hierarchical feature extraction can effectively decrease the negative interference effects from other harmonic components or strong noises and make the fault characteristics be predominant.

(2) To avoid the information loss and distortion in vectorizing the hierarchical texture features of TFT, the feature vectors from each TFI blocks are constructed into a feature tensor according the sequence of frequency band segmentation. The representation of feature tensor makes full use of abundant information on TFI and solves the "curse of dimensionality" problem in the stacking vector classification

(3) The SHTM as a tensor classifier is introduced into the pattern recognition of bearing fault states, which can effectively exploit the characteristics of features among multiple modes and the redundancy from interband correlation. The experiment results showed that the tensor classifier performs greatly better than the conventional vector-based classifiers in the rotor fault pattern recognition, especially in the case of the small size sample.

In a word, for the state identification of rotor system the proposed method can obtain high fault pattern recognition rate, good robustness to the selection of time-frequency transform, and the number of TFT segments. This promising method will contribute to machine working conditions monitoring and fault diagnosis.

\section{Competing Interests}

The authors declare that they have no competing interests. 


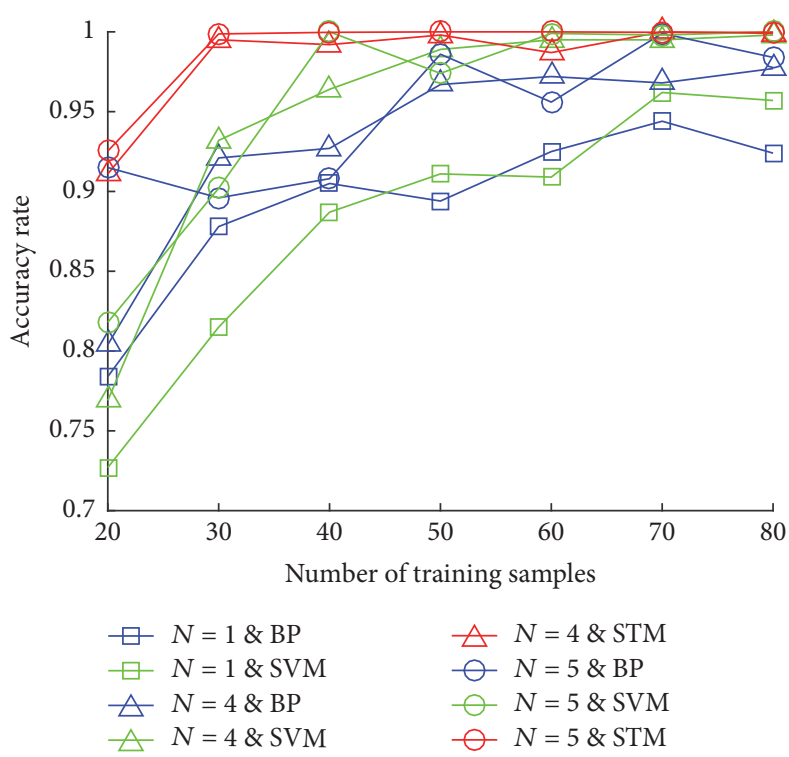

(a) STFT

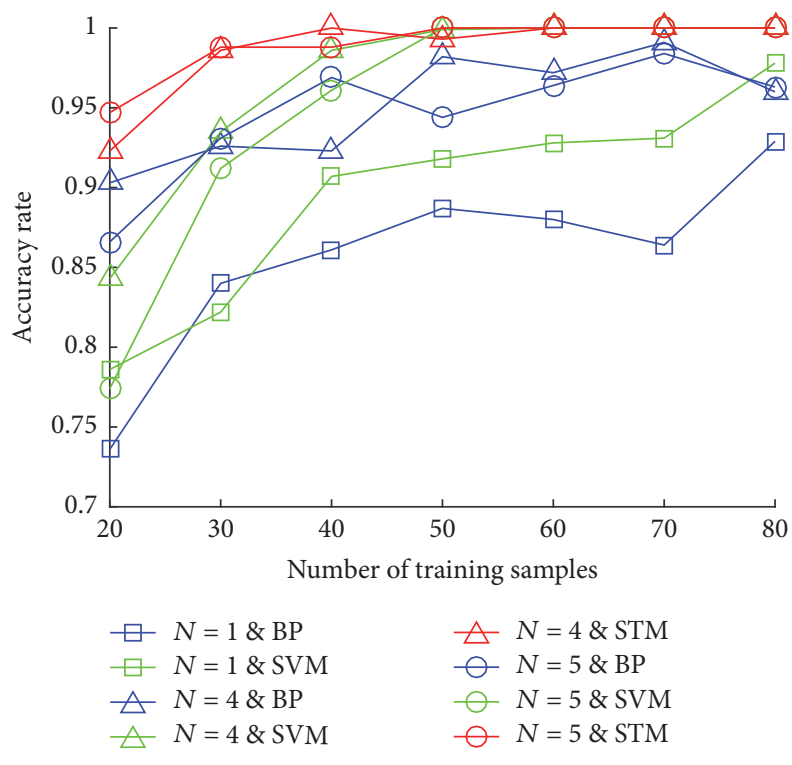

(c) CWT

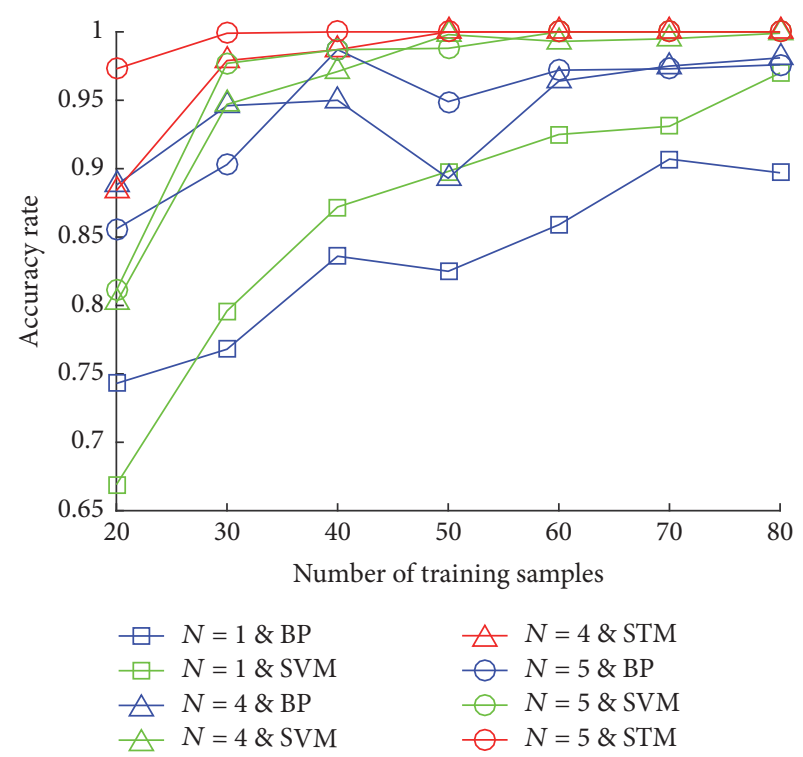

(b) S-T

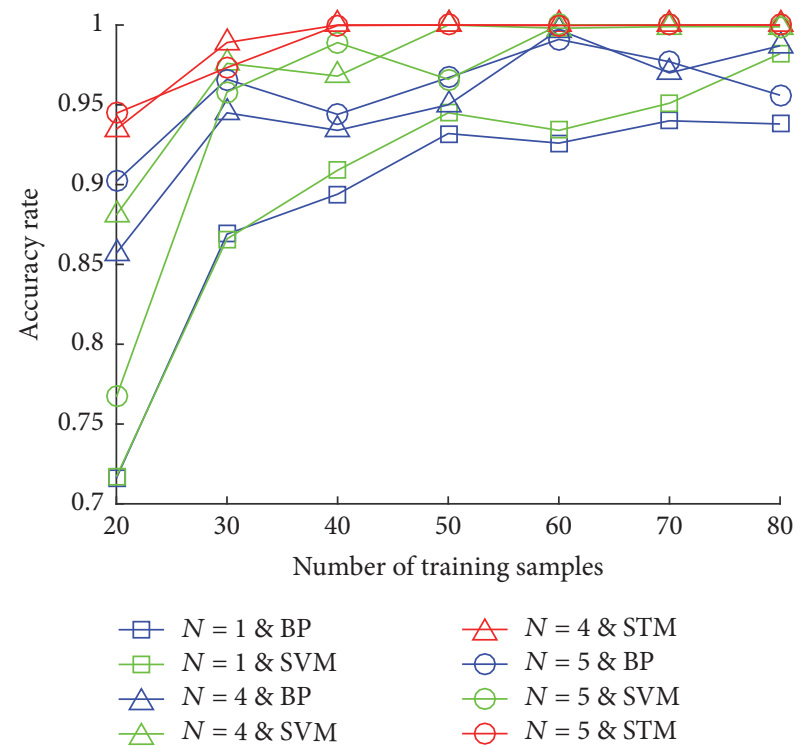

(d) $\mathrm{AOK}$

FIGURE 6: Comparison of recognition rate between hierarchical texture algorithms and traditional texture algorithms.

\section{Acknowledgments}

This work is supported by the National Natural Science Foundation Project (nos. 51475052 and 51675064), the Fundamental Research Funds for the Central Universities (no. 106112016CDJZR115502), and the China Postdoctoral Science Foundation (nos. 2016T90833 and 2015M582519).

\section{References}

[1] A. K. Jalan and A. R. Mohanty, "Model based fault diagnosis of a rotor-bearing system for misalignment and unbalance under steady-state condition," Journal of Sound and Vibration, vol. 327, no. 3-5, pp. 604-622, 2009.
[2] X. Liu, L. Bo, and H. Luo, "Smoothing localized directional cyclic autocorrelation and application in oil-film instability analysis," Journal of Sound and Vibration, vol. 368, pp. 191-201, 2016.

[3] X. Liu and L. Bo, "Identification of resonance states of rotorbearing system using RQA and optimal binary tree SVM," Neurocomputing, vol. 152, pp. 36-44, 2015.

[4] G. Badr and B. J. Oommen, "On optimizing syntactic pattern recognition using tries and AI-based heuristic-search strategies," IEEE Transactions on Systems, Man, and Cybernetics, Part B: Cybernetics, vol. 36, no. 3, pp. 611-622, 2006.

[5] S. Omachi and M. Omachi, "Fast template matching with polynomials," IEEE Transactions on Image Processing, vol. 16, no. 8, pp. 2139-2149, 2007. 
[6] M. Holden, "A review of geometric transformations for nonrigid body registration," IEEE Transactions on Medical Imaging, vol. 27, no. 1, pp. 111-128, 2008.

[7] M.-K. Hu, "Visual pattern recognition by moment invariants," IRE Transactions on Information Theory, vol. 8, no. 2, pp. 179187, 1962.

[8] A. Baraldi and F. Parmiggiani, "Investigation of the textural characteristics associated with gray level cooccurrence matrix statistical parameters," IEEE Transactions on Geoscience and Remote Sensing, vol. 33, no. 2, pp. 293-304, 1995.

[9] N. Sarkar and B. B. Chaudhuri, "An efficient differential boxcounting approach to compute fractal dimension of image," IEEE Transactions on Systems, Man and Cybernetics, vol. 24, no. 1, pp. 115-120, 1994.

[10] B. Li, P.-L. Zhang, D.-S. Liu, S.-S. Mi, G.-Q. Ren, and H. Tian, "Feature extraction for rolling element bearing fault diagnosis utilizing generalized S transform and two-dimensional nonnegative matrix factorization," Journal of Sound and Vibration, vol. 330, no. 10, pp. 2388-2399, 2011.

[11] B. Li, S.-S. Mi, P.-Y. Liu, and Z.-J. Wang, "Classification of time-frequency representations using improved morphological pattern spectrum for engine fault diagnosis," Journal of Sound and Vibration, vol. 332, no. 13, pp. 3329-3337, 2013.

[12] C. Wang, Y. Zhang, and Z. Zhong, "Fault diagnosis for diesel valve trains based on time-frequency images," Mechanical Systems and Signal Processing, vol. 22, no. 8, pp. 1981-1993, 2008.

[13] Q. Wang, Y. Zhang, Y. Zhu, and J. Yang, "Fault diagnosis of time-frequency images based on non-negative factorization and neural network ensemble," in Proceedings of the International Joint Conference on Neural Networks (IJCNN '08), pp. 607-614, Hong Kong, China, June 2008.

[14] T. Guo, L. Han, L. He, and X. Yang, "A GA-based feature selection and parameter optimization for linear support higherorder tensor machine," Neurocomputing, vol. 144, pp. 408-416, 2014.

[15] Z. Hao, L. He, B. Chen, and X. Yang, "A linear support higherorder tensor machine for classification," IEEE Transactions on Image Processing, vol. 22, no. 7, pp. 2911-2920, 2013.

[16] H. Tamura, S. Mori, and T. Yamawaki, "Textural features corresponding to visual perception," IEEE Transactions on Systems, Man and Cybernetics, vol. 8, no. 6, pp. 460-473, 1978.

[17] H. Lategahn, S. Gross, T. Stehle, and T. Aach, "Texture classification by modeling joint distributions of local patterns with Gaussian mixtures," IEEE Transactions on Image Processing, vol. 19, no. 6, pp. 1548-1557, 2010. 


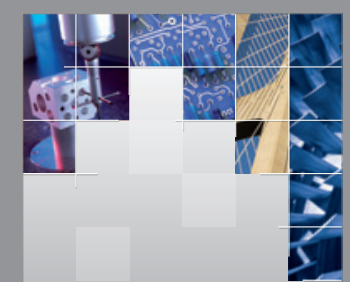

\section{Enfincering}
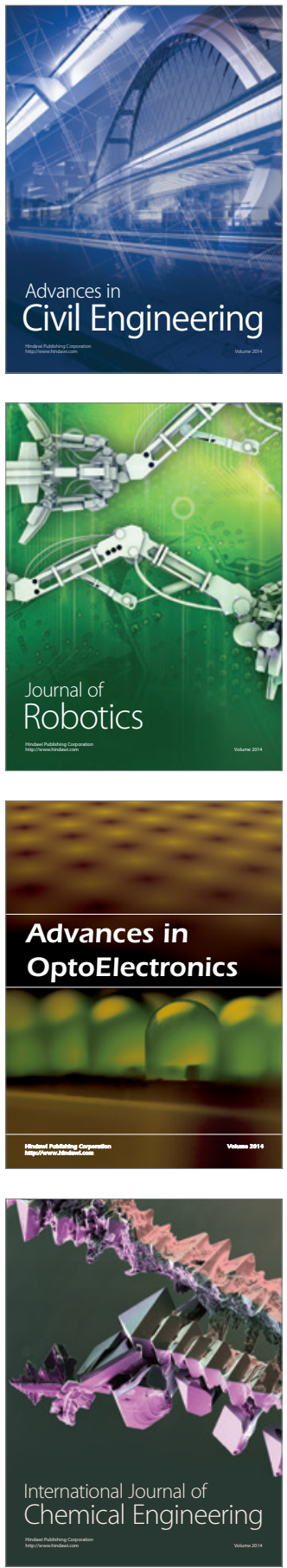

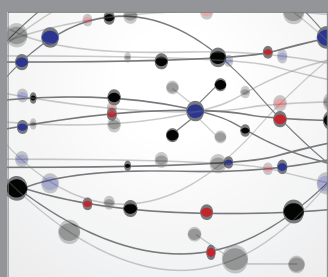

The Scientific World Journal

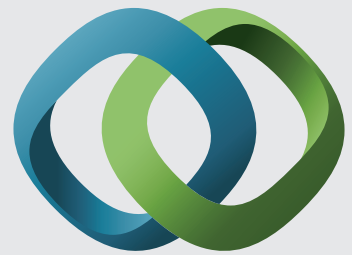

\section{Hindawi}

Submit your manuscripts at

https://www.hindawi.com
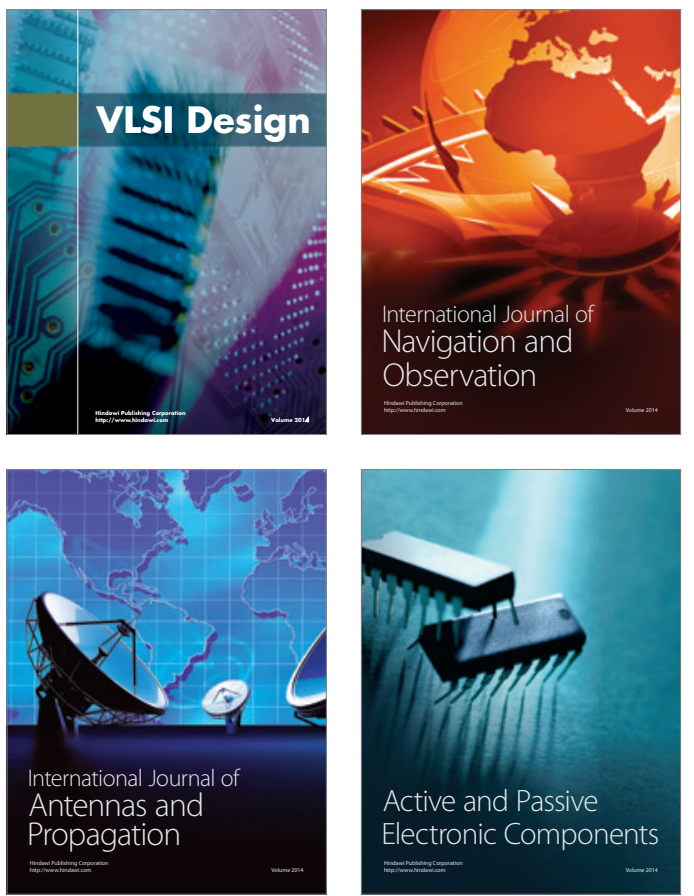
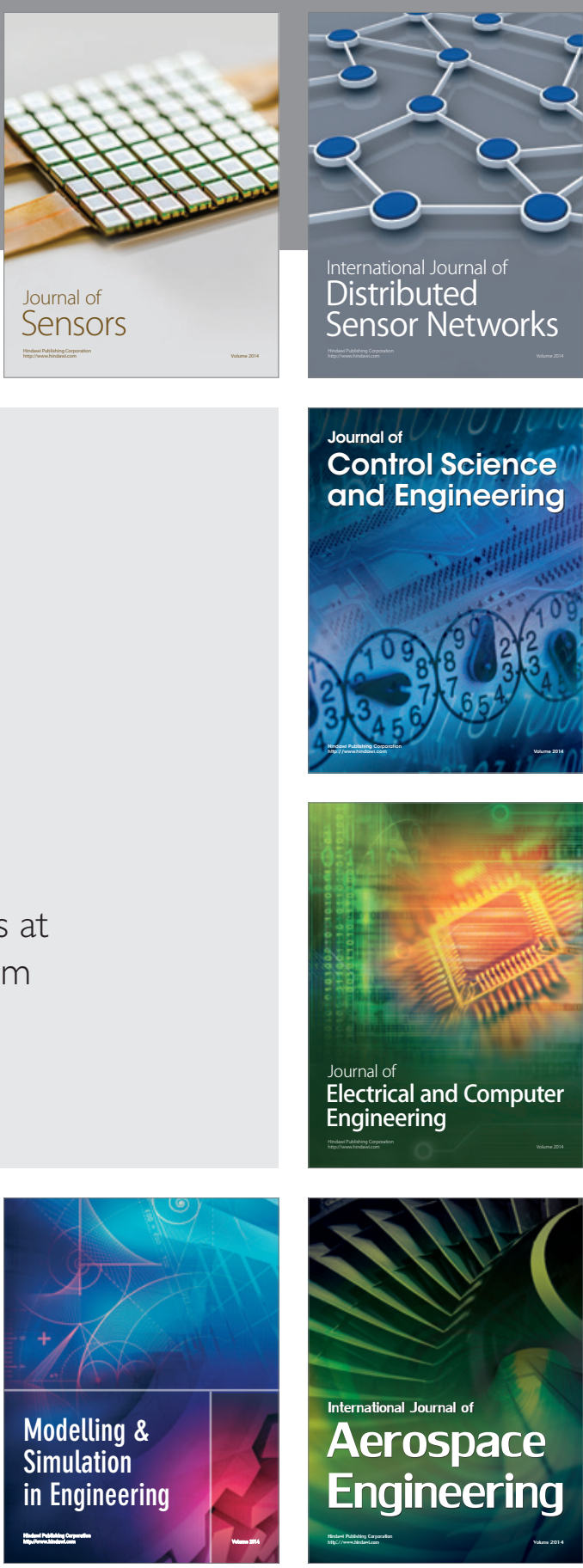

International Journal of

Distributed

Sensor Networks

$-$

Joumal of

Control Science

and Engineering
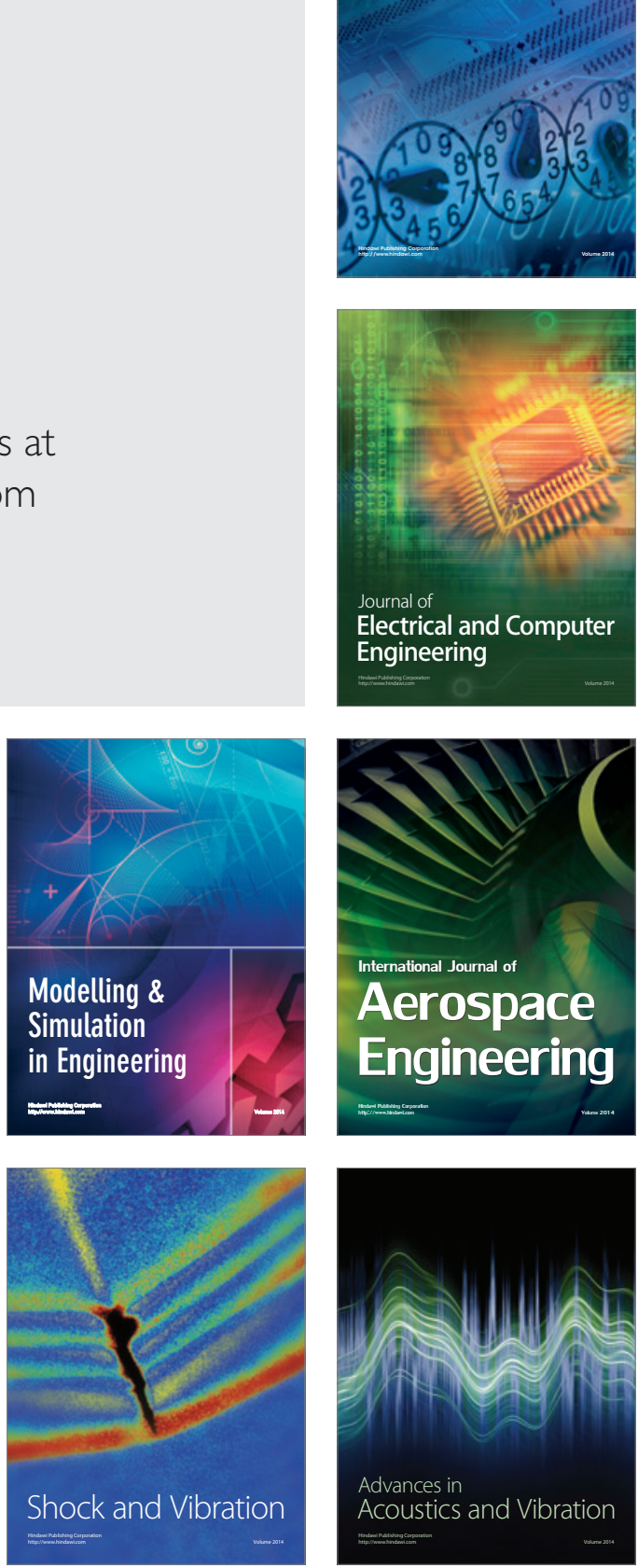